\title{
Dengue Innovation: A Sustainability Integrated Approach for Preventing and Controlling of Dengue Diseases Outbreaks via IR4.0 Technology
}

\author{
Mustafa Man ${ }^{1}$, Wan Aezwani Wan Abu Bakar ${ }^{2 *}$, Lim Chee Hwa ${ }^{3}$, Wan Nural Jawahir Wan Mohd Yusoff ${ }^{4}$ \\ ${ }^{1,3}$ Faculty of Ocean Engineering Technology and Informatics, \\ University Malaysia Terengganu (UMT), \\ 21030 Kuala Nerus, Terengganu, Malaysia. \\ mustafaman@umt.edu.my \\ ${ }^{2}$ Faculty of Informatics and Computing, Universiti Sultan Zainal Abidin, Besut Campus, 22200 Besut, \\ Terengganu. Malaysia. \\ wanaezwani@unisza.edu.my \\ ${ }^{4}$ One Team Network, 1-1B, Block 6, Jalan Pahat 15/H, Section 15, 40200 Shah Alam, Selangor. \\ limcheehwa@gmail.com
}

\begin{abstract}
Dengue is an Aedes aegypti-borne viral disease that is boosting to become worldwide serious issues. Currently, due to lack of specific treatment or vaccine against dengue, many forms of vector control remains a key strategy for dengue fever prevention. In response to the issue, this paper introduces a new Aedes trap, AedesTech Mosquitoes Home System (AMHS) that is equipped with a special trade secret formulation or also called as pheromone or Insecticide Growth Regulator (IGR) and combines with IR 4.0 technology for vector control. An IGR is a pheromone-like liquid formulation that is a chemical that serves to stimulate and have sexually attract the male and female that will attract and lure adult female mosquitoes in laying their eggs, and die afterwards. Approximately $99 \%$ of total eggs that already contaminated with the chemical do not hatch or die or go beyond the pulp level. Our sustainable approach offers for two (2) novel features. First, to monitor the collection of Aedes eggs in our MHS, we develop a mobile apps called AedesTech Apps (ATA) for Aedes eggs auto-counting and data collection are stored in cloud based server. Second, to monitor the level of liquid formulation (IGR) in AMHS, we utilize an Arduino water based sensor to real time track the IGR level status and we called as Intelligent Mosquito Home System (iMHS). Our iMHS will automatically alert the user when refill is needed. Both technologies are related to IR 4.0 that could be a new approach for preventing and monitoring of dengue outbreaks. This sustainability integrated approach is environmentally friendly and cost saving that provide the solution to the world's global issue of dengue. The results by our ATA tool indicate a satisfactory figure with only 0.01 to 0.04 percent of error rate compared to existing ICount and EggCounter tools. Meanwhile in our iMHS with water based sensor, testing results reveal that the battery lifetime solely
\end{abstract}

depends upon the setting of interval time of sending data to cloud server. Results show for a half an hour interval, battery could last for a half of a month whereas for an hour interval time set, it could last for a month.

Key words: AedesTech Mosquitoes Home System (AMHS), Intelligent Mosquito Home System (iMHS), AedesTech Apps (ATA), Insecticide Growth Regulator (IGR)

\section{INTRODUCTION}

Aedes aegypti is the main vector borne and epidemiologically diseases include dengue, Chikugunya, Zika and yellow fever [1]. It is a domestic endophilic mosquito that has expanded its habitable range in recent decades and will likely continue to spread [2]. The four (4) Aedes mosquitoes life stages are egg, larva, pupae and adult. These mosquitoes can survive and breed both inside and outside the house. The entire life cycle resumes in just 8 to 10 days. The growth from pupae into adult mosquitoes in approximately 2 to 3 days. Dengue hemorrhagic fever (DHF) or dengue fever (DF) cases are arising. Total number of dengue cases in Malaysia for 2019 to 130,101, beating the previous historic high of 120,836 cases reported in 2015 [3]. The Aedes aegypti and Aedes albopictus are two (2) major vector mosquitoes of DHF and DF. Vector control is one of the most effective approaches in combating DHF or DF. As DHF and DF can be transmitted transovarially [4-5] vector control of Aedes species in the immature stages is very important.

The researches have proved that Aedes aegypti is resistant towards organophosphate like temephos, malathion, fenthion and propoxur) [6-7], permethrin [8], DDT and dieldrin [9]. The IGR that contains a pyriproxyfen (PPF) also proved to be effective against vector mosquitoes such as aegypti [10], Culex [11-12] and Anopheles [13]. The PPF mimics a natural hormone that could disrupts their growth 
and prevent the immature mosquito to be emerged to adult. It affects the young insects and eggs as well as other insects including fleas, cockroaches, ticks, ants, carpet beetles, and mosquitoes. The Environmental Protection Agency (EPA) has registered the PPF to be used in pesticides [14].

This paper presents two (2) novel approaches in preventing and controlling dengue outbreaks. First, our AedesTech mobile Apps (ATA) that able to scan and capture images of Aedes eggs and transfer for an automated image processing system to first segregate between Aedes eggs with non-Aedes eggs and then, count its total for a further data analytic process. Second, our intelligent AedesTech Mosquito Home System (iMHS) with the embedded water-based sensor in our AedesTech Mosquito Home System (AMHS) to track for the liquid formulation, Insecticide Growth Regulator (IGR) status, equipped in our AMHS. Our approaches offer for an efficient automated counting of Aedes eggs while reducing manpower cost to check and change for IGR refill in each particular AMHS.

\section{METHODOLOGY OF DENGUE CONTROL USING AEDESTECH MOSQUITO HOME SYSTEM (AMHS)}

The design of AedesTech Mosquito Home System (AMHS) and the actual AHMS trap are shown in Figure 1, which consist of devices designed to attract Aedes mosquitoes and target container-breeding mosquitoes. AMHS is provided with an attractive water source (IGR) for gravid females in searching for its oviposition sites. Those females who has attracted to the device will be contaminated with that IGR and thus kill and eliminated its reproduction. The device is an auto-dissemination device, consisting of a regular ovitrap containing pyriproxyfen, to contaminate gravid female mosquitoes during oviposition, allowing them to disseminate the chemical to other Aedes oviposition sites. Auto-dissemination is a 'pull' and 'push' technology which allows mosquito control professionals to treat larval habitats in a timely and economical fashion [15]. The objective of the AMHS is by using the "Lure \& Kill Technology" to control \& eliminate mosquitoes during all development stages, be it as eggs, larvae, pupae or adults, and thus to reduce the overall mosquito population.

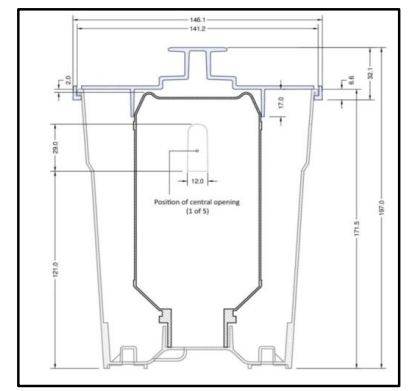

(a) AMHS Interior design

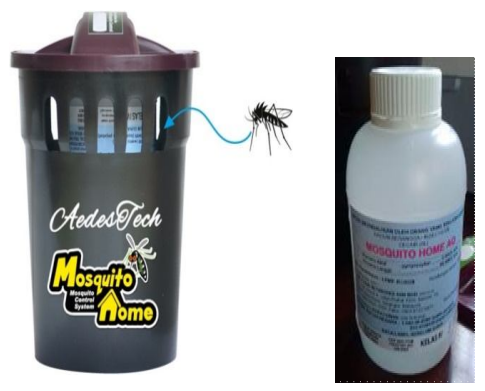

(b) Real AMHS and its IGR
Figure 1: AMHS Interior Design and the Real Physical AHMS with the Water Based Attractant Refill

\section{DEVELOPMENT OF AEDESTECH APPS FOR MOSQUITOE EGGS COUNTING}

Through this project, the mobile AedesTech Apps (ATA) is developed to collect data related to Aedes mosquito eggs directly from AMHS and stored in a cloud database. For this purpose, a rectangular piece of tissue is clipped to similar size of transparency slides and both materials are circularly attached around the inner part of AMHS. The reason is to allow for a slowly absorption of the IGR into the tissue that allow for breeding process of female Aedes mosquitoes. The tissue is then taken out to be scanned by ATA and transfer the digitized images to the Excel file for an auto counting for Aedes eggs via MATLAB AutoCountMe [16] system. Through this system, the exact images of Aedes eggs are identified from non-Aedes eggs through digital image processing technology to be developed. A new algorithm will be proposed to expedite the process of counting the mosquito eggs directly using only mobile devices or mobile phones either offline or online. Refer to Figure 2 for the overall project framework. The large data from various installations of the whole AMHS in geographically apart locations are then collected and compiled into the central cloud database for further numerous data mining and forecasting analysis. Example of the analysis would be to projection of the mosquito population rate for a region or location and it can extend to the parties for follow-up actions and comparative results that can be utilized for the manufacture decision [17].

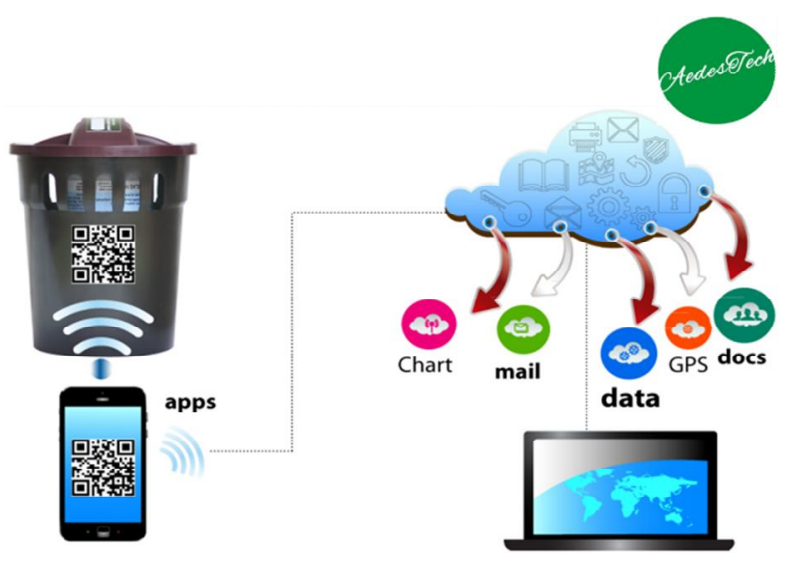

Figure 2: ATA Development Framework

The unique feature that ATA offers is for an in-situ data collection. All digitized images of an Aedes eggs are collected and auto-counting using this apps. Using ATA, several data mining tools could be retrieved based on image captured. Figure 3 illustrates and ATA algorithm and Figure 4 depicts an ATA user interface.

1. Start

2. Login id and password (need to turn on location)

3. Pot or MHS trap Setup and labeling using QR or Bar 
Code

4. Setup and configure the location of the trap using Global Positioning System (GPS)

5. Click the camera inside the apps and scan the QR-Coded

6. $\quad$ select the image

7. click scan and

8. count the Aedes eggs.

9. Save in cloud or in the mobile if no internet coverage 10. Statistical and data visualization by each AMHS trap will visualize automatically.

\section{Finish}

Figure 3: ATA Algorithms
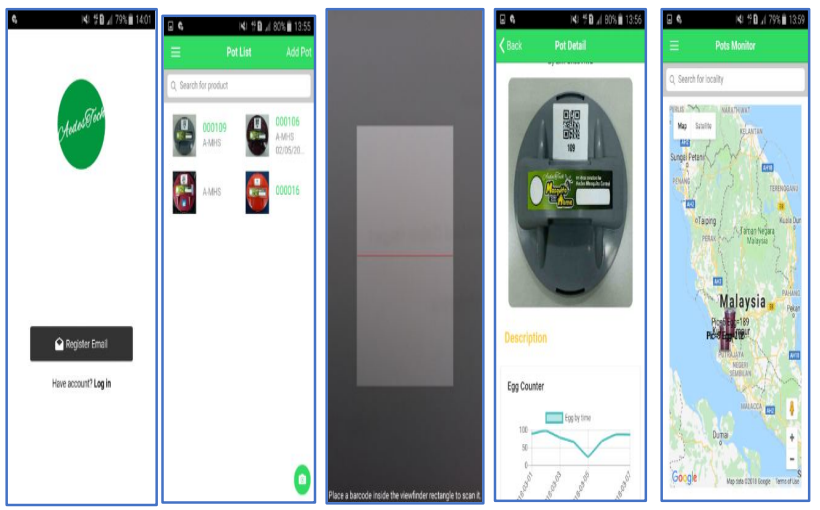

Figure 4: ATA User Interfaces

\section{DEVELOPMENT OF IMHS: INTELLIGENT MOSQUITOE HOME SYSTEM USING WATER BASE SENSOR}

The iMHS design framework relies upon 3-tier architecture. The Presentation tier is User Interface part that interfacing with client. The Application tier is the middle part where logic operations are undertaken to ease of communication between client and data layers. Then, the Data tier refers to actual database that includes insert, delete, update and get data from/to database operations.

\subsection{Hardware Component}

The three (3) basic components of iMHS devices include the microcontroller, a water level sensor to perform the intended task and the source of power, an AA battery. The ESP8266 microcontroller [18-19], a Wi-Fi embedded microcontroller that utilizes $2.4 \mathrm{GHz}$ frequency. Then, an Arduino water level sensor [20], an effective water-based sensor to indicate for water level drop status that shows signals conversion of water to analog. The Arduino development board simulates the value to give the readings of water level alarm. Lastly, the power source that refers to the AA battery [20] or the standard size dry battery. It composes of a single electrochemical use in a disposable battery or a rechargeable battery. A prescription of all components is shown in Table 1 and iMHS circuit board connection is illustrated in Figure 5. The actual implementation of our Arduino water level sensor in AMHS is depicted in Figure 6. The iMHS adopts MySQL Database Management System (DBMS) and PHP 7 for page scripting.

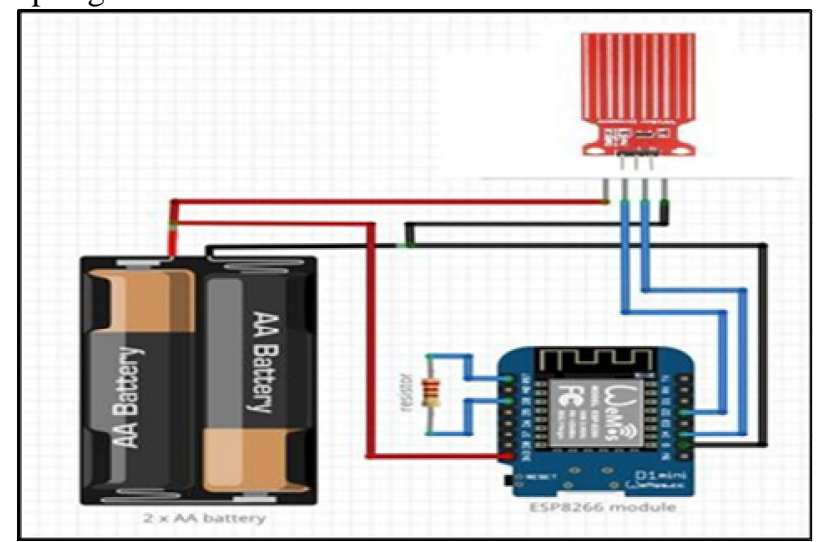

Figure 5: iMHS Circuit Board Design

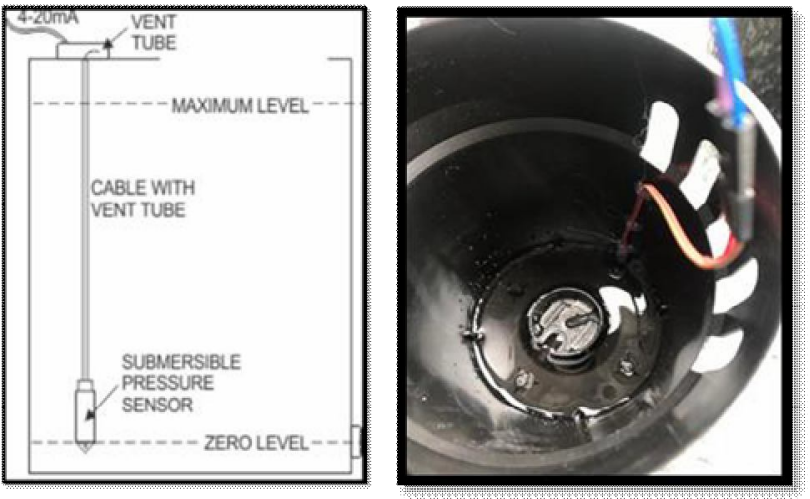

Figure 6: Arduino water level sensor in AMHS

\section{DISCUSSION}

\subsection{Result for ATA}

Our running dataset is extracted from [21] together with their ICount tool. We set five (5) macro images (from camera) and five (5) micro image from microscopic (as shown in Figure 7). Our ATA method is compared to ICount [21] and EggCounter [22] which are free downloadable. ImageJ (from manual counting) is set as a benchmark for an accuracy validation of each tool. Table 1 shows the comparison results between ATA, ICount and ImageJ. The EggCounter could not detect any single egg.

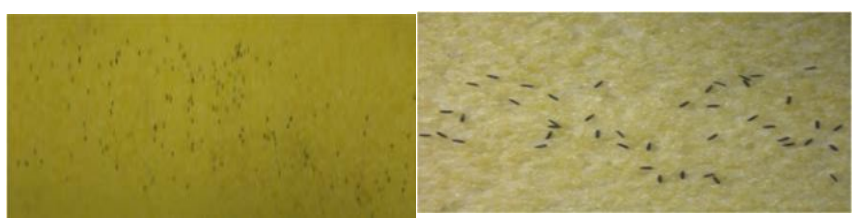

(a) Macro Image

(b) Micro Image

Figure 7: Macro and Micro Image of Aedes Eggs 
The ICount requires most user intervention and the results are depending upon setting parameters, thus could affect the estimation process. In contrast to ICount, the ATA requires only the loading of the image to automatically count eggs. The results from ATA are satisfactory with the small error rate of 0.01 to 0.04 percent.

Table 1: Evaluation on ImageJ, ATA method, ICount and EggCounter on Macro Images

\begin{tabular}{lcccccc}
\hline Dataset ImageJ & $\begin{array}{c}\text { ATA } \\
\text { Method }\end{array}$ & $\begin{array}{c}\text { \% } \\
\text { Error }\end{array}$ & ICount & $\begin{array}{c}\text { \% } \\
\text { Error }\end{array}$ & $\begin{array}{l}\text { Egg } \\
\text { Count } \\
\text { er }\end{array}$ & $\begin{array}{c}\text { \% } \\
\text { Error }\end{array}$ \\
\hline $\begin{array}{l}\text { IMG_22 } \\
\text { 5bis }\end{array}$ & 155 & 0.01 & 146 & 0.05 & 0 & 100 \\
\hline $\begin{array}{l}\text { IMG_22 } \\
\text { 53bis }\end{array}$ & 479 & 0.04 & 482 & 0.01 & 0 & 100 \\
\hline $\begin{array}{l}\text { IMG_22 } \\
\text { 56bis }\end{array}$ & 348 & 0.01 & 344 & 0.01 & 0 & 100 \\
\hline $\begin{array}{l}\text { IMG_22 } \\
\text { 57bis }\end{array}$ & 213 & 0.04 & 315 & 0.03 & 0 & 100 \\
\hline $\begin{array}{l}\text { IMG_22 } \\
\text { 67bis }\end{array}$ & 358 & 0.01 & 334 & 0.07 & 0 & 100 \\
\hline
\end{tabular}

\subsection{Result of iMHS}

In iMHS, the issue arise regarding the battery power source lifetime. We run several tests to Arduino water based sensor with the AA battery in two different interval of data sent to cloud database. The results are as depicted in Table 2 . Implementation results reveal that the battery lifetime solely depends upon the setting of interval time of sending data to cloud server. Changing in interval time proportionately resulting in changing the lifetime of battery power energy. The iMHS overall results show for a half an hour interval, battery could last for a half of a month whereas for an hour interval time set, it could last for a month.

Table 2: Results of battery lifetime in different interval

\begin{tabular}{l|ll}
\hline $\begin{array}{l}\text { Interval time (in second) } \\
\text { of Data Send }\end{array}$ & $\begin{array}{l}\text { Battery lifetime (in } \\
\text { month) } \\
\text { (AA Battery) }\end{array}$ \\
\hline 1800 & 0.5 \\
\hline 3600 & 1 \\
\hline
\end{tabular}

The Arduino water level sensor reads only low (blue), medium (yellow) and high (red) status (as depicted in Figure 8). For each status, different distances in centimeter $(\mathrm{cm})$ have been set to read in the sensor device. The different iMHS device locations are represented through its longitude and latitude, whereas Google map will redirect into exact longitude and latitude location detail.

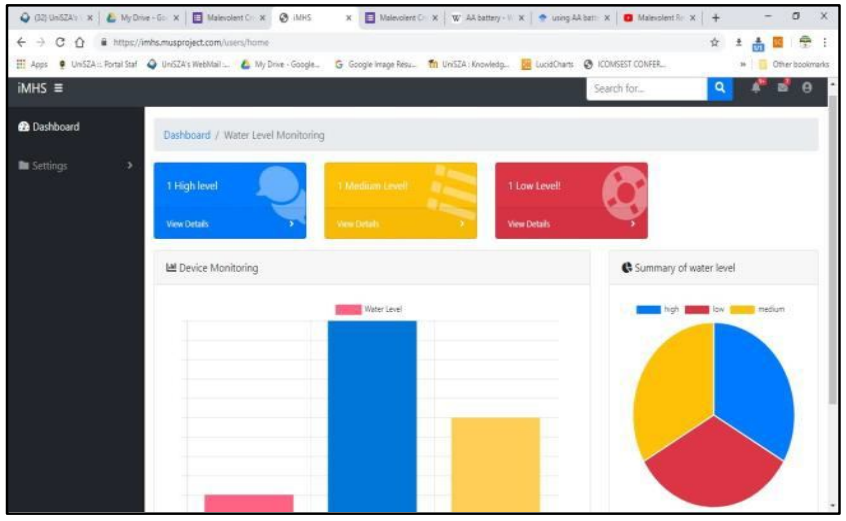

Figure 8: User interface of iMHS

\section{CONCLUSION}

The development of AMHS, Aedestech Apps (ATA) and iMHS is considered to successfully embed the Internet of Things (IOT) technology for our dengue innovation project related to Industrial Revolution (IR) 4.0. This research collaboration has transferred the knowledge and technology between universities as well as its industry partner. As a recapitulation, AMHS offers for effective alternative in controlling dengue. The ATA provides effective way in getting the input images of Aedes egg to further process of auto-counting of Aedes eggs send to cloud storage for extensive analysis. And lastly with our iMHS, the cost for labor to manually check and track for the emptied IGR is significantly reduced and cut-off through the real-time IGR status visualization. The main objective of this project for reducing manpower usage in controlling the dengue disease at the national as well as international level. The concept of auto-detection device can be applied for other industry domain that might as well create new business opportunities in the near future.

\section{ACKNOWLEDGEMENT}

We thank all our research institutions-industry collaborators i.e. UniSZA, UMT, UniKL and One Team Networks (OTN) Sdn. Bhd. under matching grant, UniSZA/2018/PKP/01 with project code R0047-R001. A sincere gratitude goes to the MHS co-founder, One Team Network Sdn. Bhd. As well as for supporting our work in reviewing for spelling errors and synchronization consistencies and also for the constructive comments and suggestions.

\section{REFERENCES}

1. H. J. Beaty, B. J., Rowland, M., Scott, T. W., \& Sharp, B. L. "The Innovative Vector Control Consortium: improved control of mosquito-borne diseases." Trends in parasitology, 22(7), 2006. 308-312.

https://doi.org/10.1016/j.pt.2006.05.003 
2. A. Buchman, G. Stephanie, L. Ming, A. Igor, L. Shin-Hang, W. Shin-Wei, C. Chun-Hong. "Broad Dengue Neutralization in Mosquitoes Expressing an Engineered Antibody." CELL-D-19-01515 (2019). https://doi.org/10.2139/ssrn.3398490

3. C. M. Seng, and N. Jute. "Breeding of Aedes aegypti (L.) and Aedes albopictus (Skuse) in urban housing of Sibu town, Sarawak". Southeast Asian Journal of Tropical Medicine and Public Health. 1994, Sep;25:543.

4. L. Han Lim, I. Mustafa Kamal, and A. Rohani "Does transovarial transmission of dengue virus occur in Malaysian Aedes aegypti and Aedes albopictus?" Southeast Asian Journal of Tropical Medicine \& Public Health 28.1 (1997): 230-232.

5. H. L. Lee, "Environmental friendly approaches to mosquito control." Mosquitoes and Mosquito-borne Diseases. Ng, FSP and Yong, HS (eds.). Kuala Lumpur: Academy of Science Malaysia (2000): 223-233.

6. World Health Organization. Dengue haemorrhagic fever: diagnosis, treatment, prevention and control. World Health Organization, 1997. George G. P., and Taylor C. E. "Genetic and biological influences in the evolution of insecticide resistance." Journal of economic entomology 70.3 (1977): 319-323.

https://doi.org/10.1093/jee/70.3.319

7. C. A. Malcolm, and R. J. Wood. "Location of a gene conferring resistance to knockdown by permethrin and bioresmethrin in adults of the BKPM3 strain of Aedes aegypti." Genetica 59.3 (1982): 233-237.

8. World Health Organization. Test procedures for insecticide resistance monitoring in malaria vectors, bio-efficacy and persistence of insecticides on treated surfaces: report of the WHO informal consultation, Geneva, 28-30 September 1998. No. WHO/CDS/CPC/MAL/98.12. Geneva: World Health Organization, 1998.

9. P. Y. Loh, and H. H. Yap. "Laboratory Studies on the Efficacy and Sublethal Effects of an Insect Growth Regulator, Pyriproxyfen (S-31183) Against Aedesaegypti (Linnaeus)". 1989.

10. K. K.amimura, and R. Arakawa "Field evaluation of an insect growth regulator, pyriproxyfen, against Culex pipiens pallens and Culex tritaeniorhynchus." Medical Entomology and Zoology 42.3 (1991): 249-254. https://doi.org/10.7601/mez.42.249

11. C. H. Schaefer, and F. S. Mulligan. "Potential for resistance to pyriproxyfen: a promising new mosquito larvicide." Journal of the American Mosquito Control Association 7.3 (1991): 409-411.

12. T. Okazawa. "Research \& Reports." Journal of the American Mosquito Control Association 7.4 (1991): 604-607.

13. Pyriproxyfen, General Fact Sheets. Available: http://npic.orst.edu/factsheets/pyriprogen.html

14. I. Unlu, D. S. Suman, Y. Wang, K. Klingler, A. Faraji, and R. Gaugler "Effectiveness of autodissemination stations containing pyriproxyfen in reducing immature
Aedes albopictus populations." Parasites \& vectors 10, no. 1 (2017): 139.

https://doi.org/10.1186/s13071-017-2034-7

15. W. Y. W. N. Jawahir, M. Mustafa, M. S. Hitam, A. A. K. A. Hamid, E. A. Awalludin, and W. A. W. A. Bakar. "Wavelet-based Auto-Counting Tool of Aedes Eggs." In Proceedings of the 2018 International Conference on Sensors, Signal and Image Processing, pp. 56-59. ACM, 2018.

16. M. Mustafa, W. A. W. A. Bakar, W. N. J. W. M. Yusoff, and M. A. M. Nor. "AedesApps: Image Processing Algorithm on Aedes Eggs Auto-Counting Mobile Apps."

17. NodeCMU Documentation. Available: https://nodemcu.readthedocs.io/en/master/

18. P. Patnaikuni, D. R. "A Comparative Study of Arduino, Raspberry Pi and ESP8266 as IoT Development Board." International Journal of Advanced Research in Computer Science 8, no. 5 (2017).

19. AA Battery. Available: https://en.wikipedia.org/wiki/AA_battery.

20. G. P. Nayaka, KV. Pai, G. Santhosh, J. Manjanna, 'Dissolution of cathode active material of spent Li-ion batteries using tartaric acid and ascorbic acid mixture to recover Co. Hydrometallurgy", 2016 May 1;161:54-7. https://doi.org/10.1016/j.hydromet.2016.01.026

21. J. Gaburro, J.B. Duchemin, P.N. Paradkar, S. Nahavandi, and A. Bhatti, "Assessment of ICount software, a precise and fast egg counting tool for the mosquito vector Aedes aegypti”. Parasites \& vectors, 2016. 9(1), p.590.

22. A. Mollahosseini, M. Rossignol, C. Pennetier, A. Cohuet, A. dos Anjos, F. Chandre, and H.R. Shahbazkia, "A user-friendly software to easily count Anopheles egg batches". Parasites \& vectors, 2012, 5(1), p.122.

https://doi.org/10.1186/1756-3305-5-122 